\title{
Evaluation of the efficacy and tolerability of miglitol in Chinese patients with type 2 diabetes mellitus inadequately controlled by diet and sulfonylureas
}

\author{
Sheng-Hwu Hsieh • Kuang-Chung Shih • \\ Chien-Wen Chou • Chih-Hsun Chu
}

Received: 6 January 2010/Accepted: 31 August 2010/Published online: 21 October 2010

(C) The Author(s) 2010. This article is published with open access at Springerlink.com

\begin{abstract}
The objective of this study is to examine the efficacy and tolerability of miglitol with respect to improving glycemic control in Chinese patients with type 2 diabetes mellitus inadequately controlled by diet and sulfonylurea treatment. This was a randomized, double-blinded, placebo-controlled, multicenter study. A total of 105 patients were randomized to receive 24 weeks of treatment with miglitol ( $n=52$; titrated from $50 \mathrm{mg}$ to $100 \mathrm{mg} 3$ times daily) or placebo $(n=53)$. Concomitant sulfonylurea treatment and diet remained unchanged. The primary endpoint was change in glycated hemoglobin (HbAlc) from baseline at 24 weeks. Secondary endpoints were changes in fasting plasma glucose (FPG), postprandial
\end{abstract}

Kuang-Chung Shih and Sheng-Hwu Hsieh contributed to this paper equally.

S.-H. Hsieh ( $₫)$

Division of Endocrinology and Metabolism,

Chang Gung Memorial Hospital, Chang Gung University

College of Medicine, 5, Fu-Shing St, Kwei-Shan,

Taoyuan 333, Taiwan, ROC

e-mail: cgmhsh4821@yahoo.com.tw

\section{K.-C. Shih $(\bowtie)$}

Division of Endocrinology and Metabolism,

Tri-Service General Hospital, National Defense Medical Center,

No. 325, Sec. 2, Chenggong Rd, Neihu District,

Taipei City 114, Taiwan, ROC

e-mail: shihkc@totalbb.net.tw

C.-W. Chou

Division of Endocrinology and Metabolism,

Chi Mei Medical Center, Tainan, Taiwan

C.-H. Chu

Division of Endocrinology and Metabolism,

Kaohsiung Veterans General Hospital, Kaohsiung, Taiwan plasma glucose (PPG), and postprandial serum insulin (PSI). The miglitol treatment group showed significantly greater reductions in $\mathrm{HbA1c}$ and PPG levels compared with the placebo group. With respect to adverse events, abdominal discomfort, diarrhea, and hypoglycemia occurred with similar frequency in both groups. Results of this study indicate that miglitol significantly improves metabolic control in Chinese patients with type 2 diabetes mellitus. Miglitol is safe and well tolerated, with the exception of abdominal discomfort. Therefore, miglitol may be a useful adjuvant therapy for Chinese patients with type 2 diabetes mellitus inadequately controlled by diet and sulfonylurea treatment.

Keywords $\alpha$-Glucosidase inhibitors · Miglitol .

Type 2 diabetes mellitus

\section{Introduction}

Diabetes mellitus, a rapidly growing health problem in many countries, is an important cause of morbidity and mortality. According to the US Centers for Disease Control and Prevention, approximately 14.7 million people in the United States were diagnosed with diabetes as of 2004, with type 2 diabetes accounting for approximately $90 \%$ of those cases [1]. Type 2 diabetes has resulted in an extremely large and growing economic burden. Despite the availability of effective diabetes-specific therapies, achievement of glycemic goals by patients is far from adequate in the United States. Less than half of adults with diabetes are reported to attain a glycated hemoglobin (HbA1c) level of $<7 \%$ [2]. From 1960 to 1988 in Taiwan, mortality ascribed to diabetes increased 6.3-fold [3]. In another study from Taiwan, with a total of $1,124,348.4$ person-years of follow-up, 
43,888 patients with diabetes died, and the crude mortality rate was 39.0/1,000 person-years [4].

Maintaining a normal plasma glucose level is key for reducing the risk of developing complications of diabetes [5]. Current recommendations from the UK Prospective Diabetes Study Group emphasize lifestyle management, diet, and exercise as the first-line approach, followed by therapy with oral antidiabetic drugs, administered alone or in combination [5]. Recent reviews of the literature confirm the salutary effects of exercise in individuals with type 2 diabetes $[6,7]$. Other standard therapies include the use of peroxisome proliferator-activated receptor (PPAR)-gamma agonists (thiazolidinediones), which decrease levels of glycated hemaglobin, fasting plasma glucose, insulin, and free fatty acids in patients with type 2 diabetes [8,9], and insulin, together with metformin, which acts predominantly to inhibit hepatic glucose production, or with insulin secretagogues [10, 11]. Antidiabetic drug monotherapy eventually necessitates the use of increasing dosage and/or a second antidiabetic medication because type 2 diabetes worsens over time as a result of declining pancreatic $\beta$-cell function [12].

The class of $\alpha$-glucosidase inhibitors has a unique mode of action. These drugs block oligosaccharide catabolism, delay carbohydrate digestion and absorption, and smooth and lower postprandial plasma glucose (PPG) peaks [13, 14]. Miglitol is the first pseudomonosaccharide $\alpha$-glucosidase inhibitor derived from 1-deoxynojirimycin and is structurally a glucose analogue [15]. Its efficacy, in monotherapy [16] or in combination with sulfonylureas [17], as a glucose-lowering agent in Chinese patients with type 2 diabetes has not been determined in clinical studies. The aim of this study was to investigate the efficacy and tolerability of miglitol in combination with sulfonylureas, compared to sulfonylurea monotherapy, for the improvement of glycemic control in Chinese outpatients with type 2 diabetes mellitus inadequately controlled by diet and sulfonylurea treatment.

\section{Patients and methods}

\section{Patients}

The study design was a randomized, double-blinded, placebo-controlled, multicenter comparison of miglitol treatment compared with placebo administration over a 24-week period. Patients with a confirmed diagnosis of type 2 diabetes mellitus whose previous treatment with diet and sulfonylureas had proved inadequate according to medical chart monitoring were recruited at 4 medical centers in Taiwan. Inclusion criteria included age $>20$ years; fasting plasma glucose (FPG) concentration of
$100 \mathrm{mg} / \mathrm{dL}$ to $240 \mathrm{mg} / \mathrm{dL} ; \mathrm{HbA} 1 \mathrm{c}$ value of $6.5 \%$ (based on the glycemic goal for adults of $\leq 6.5 \%$ as specified by the Diabetes Association of Taiwan) to $10.0 \%$; history of uncontrolled type 2 diabetes mellitus despite prior nutrition therapy; and stable dosing with a sulfonylurea for at least 8 weeks before randomization.

Exclusion criteria included the following: suggested diagnosis of type 1 diabetes mellitus; active insulin therapy, known lactose intolerance, or treatment with medication that significantly alters gastrointestinal motility and/ or absorption; concomitant glucocorticoid therapy, other medication affecting glucose homeostasis, or treatment with investigational drugs; serum transaminase level $>2.5$ times the upper normal limit or serum creatinine level $>1.5 \mathrm{mg} / \mathrm{dL}$; presence of significant disease or condition (including emotional disorder or substance abuse) that would likely alter the course of diabetes or the patient's ability to complete the study; documented gastrointestinal disease associated with marked disorder of digestion or absorption, or condition that may worsen as a result of increased gas formation in the intestine; pregnant or lactating women or women of childbearing age without a medically approved method of contraception; and patients participating in another clinical trial within 90 days of screening.

This study was conducted in accordance with the European Community guidelines for Good Clinical Practice and the Declaration of Helsinki and its amendments. The protocol was approved by the corresponding Joint Institutional Review Board or the Department of Health and Ethics Committee of each investigational site. Written informed consent was obtained from each patient.

\section{Study design and drug regimen}

Patients with uncontrolled diabetes despite nutrition therapy and sulfonylurea treatment were assigned to a 2-week baseline work-up and dietary run-in period. During this period, demographic data were obtained, and vital signs and routine laboratory variables were measured. To confirm adherence to nutrition therapy, patients were instructed to complete a 3-day diet record before visit 2. Eligible patients were randomized to receive miglitol (Migbose; Standard Chem. \& Pharm. Co., Ltd.) $50 \mathrm{mg} 3$ times daily for 12 weeks, titrated to $100 \mathrm{mg} 3$ times daily for 12 weeks, or placebo. After randomization, patients were instructed to complete a 3-day diet record before each visit. Patients were asked to adhere to a dietary plan tailored to their energy requirements and metabolic control, according to current American Diabetes Association recommendations: carbohydrates up to $60 \%$, fat $<30 \%$, and protein 12-20\%. Concomitant sulfonylurea treatment remained unchanged throughout the study. 
Patients were instructed to take 1 miglitol or placebo tablet with the first mouthful of food at each of 3 main daily meals. Drug compliance was determined by tablet count at each visit. After randomization (week 0), patients were assessed at weeks 4, 8, 12, 16, 20, and 24. A physical examination, assessment of adverse events, dietary counseling, and measurement of HbA1c were carried out at each visit. All secondary efficacy variables and routine laboratory variables were measured at baseline obtained before week 0 during the run-in period and at week 24 . In cases of premature termination, routine laboratory variables were measured at the last visit. Dose titration at week 12 was performed at the discretion of the investigator. Patients with good tolerance to miglitol $50 \mathrm{mg} 3$ times daily were titrated to $100 \mathrm{mg} 3$ times daily. Patients with unsatisfactory, but acceptable, tolerance to the treatment were maintained at $50 \mathrm{mg} 3$ times daily. Patients unable to tolerate the treatment were discontinued from the study. Patients reported to the study station between 08:00 and 08:30 AM after a 12-h fast. After emptying the bladder, body height and weight were measured, and body mass index (BMI) was calculated as body weight $(\mathrm{kg})$ divided by height squared $\left(\mathrm{m}^{2}\right)$. An electrocardiogram was performed for all patients to evaluate cardiac function. The antecubital vein of the arm was cannulated for blood sampling. Baseline or fasting blood samples were obtained after approximately $10 \mathrm{~min}$ of rest after cannula placement.

\section{Laboratory methods}

Venous blood samples were placed into individual tubes with ethylenediaminetetraacetic acid. Aliquots of serum and plasma were stored at $-80^{\circ} \mathrm{C}$. Samples from each patient were measured in the same assay to reduce interassay variation. Hematology, biochemical assays (sodium, potassium, serum creatinine, alkaline phosphatase, alanine aminotransferase [ALT], aspartate aminotransferase [AST], total protein), and lipid assays (triglyceride, total cholesterol, low-density lipoprotein cholesterol [LDL-C], highdensity lipoprotein cholesterol [HDL-C]) were carried out by routine automated methods. Plasma glucose was detected by the glucose oxidase method with a 2300 STAT glucose analyzer (Yellow Springs Instrument Inc., Yellow Springs, OH). Serum insulin was determined by microparticle enzyme immunoassay with an AxSYM system (Abbott Laboratories, Abbott Park, IL). Measurement of HbA1c was performed with a DCA 2000 analyzer (Bayer Diagnostics, Elkhart, IN).

\section{Efficacy and safety assessments}

Intent-to-treat (ITT) analysis was performed for assessment of efficacy. Patients were included in the ITT analysis if they had efficacy data at baseline (week 0) and at least 1 postbaseline efficacy measurement. The primary efficacy variable was HbAlc concentration. The endpoint was defined as the last available measurement. Secondary efficacy variables included FPG, PPG, and postprandial serum insulin (PSI). Venous blood for postprandial measurements was taken $2 \mathrm{~h}$ after a standard breakfast.

Patients were included in the safety analysis if they had taken at least 1 dose of medication and had at least 1 postbaseline safety measurement. The baseline for safety analysis was defined as measurements taken at visit 1 (week 2 ), and the endpoint was defined as the last measurements taken at visit 8 (week 24). Safety variables were analyzed descriptively. All adverse events were defined according to the Coding Symbols for a Thesaurus of Adverse Reaction Terms (COSTART) glossary and body system categories (http://hedwig.mgh.harvard.edu/biostatistics/files/costart. html).

Safety and tolerance were assessed primarily from spontaneously reported adverse events and described by the patients at each visit, with special attention to the severity of hypoglycemia; occurrences of symptoms suggestive of hypoglycemia were recorded in the patient's diary. These symptoms were rated as grade 1 (mild and transient), grade 2 (transient inability to pursue usual activities), grade 3 (need for external assistance), or grade 4 (need for medical assistance). Hypoglycemia was defined as at least 1 episode of symptoms suggestive of hypoglycemia during the study period. Other adverse events were also recorded in the patient's diary. Serious adverse events were defined as events resulting in persistent or significant disability or incapacity, new hospitalization or prolongation of current hospitalization, severe hypoglycemia, and life-threatening events or death. Acute intoxication, important medical events, and pregnancy were considered serious adverse events.

\section{Statistical analysis}

Data are shown as mean \pm standard deviation (SD) for continuous variables and as $n(\%)$ for categorical variables for demographics and adverse events follow-up. Data are shown as mean \pm standard error for change from baseline for primary efficacy endpoints. The last observation carried forward (LOCF) approach was used for evaluation of primary efficacy endpoints in the ITT population. For comparison of baseline demographics and change in efficacy endpoints from baseline, a 2-sample $t$ test was performed for continuous variables, and chi-square or Fisher exact test was performed for categorical variables. Nonparametric Wilcoxon rank-sum test was also performed if the continuous data were not normally distributed. Data analysis was 
performed with SAS version 9.0 (SAS Institute Inc., Cary, NC). Differences were considered statistically significant at $P<0.05$.

\section{Results}

Patient disposition is detailed in Fig. 1. A total of 138 patients with type 2 diabetes mellitus inadequately controlled by diet and sulfonylurea treatment were screened. A total of 105 patients were eligible for randomization; 52 were assigned to receive miglitol treatment, and 53 were assigned to receive placebo. Efficacy endpoints were analyzed in the ITT population, regardless of protocol compliance, and adverse events were followed-up in the safety population. Of the 105 patients, 100 (49 in the miglitol group and 51 in the placebo group) comprised the ITT population; 5 patients failed to return for postclinical assessment. All 105 randomized patients received treatment and were followed up for safety.

Baseline demographic and clinical characteristics of patients in the ITT population, listed by treatment group, are presented in Table 1. There were no significant differences in demographic or other baseline characteristics between the miglitol and placebo groups. Table 2 shows results for changes from baseline for the efficacy variables HbAlc, FPG, PPG, and PSI at week 24 in the ITT population. The change in $\mathrm{HbA} 1 \mathrm{c}$ from baseline for the miglitol group was $-0.85 \% \pm 0.12 \%$ compared to $-0.19 \% \pm$ $0.11 \%$ for the placebo group $(P<0.001)$. There was also a significant difference in the change in PPG between groups
$(P<0.001)$. No significant difference in change in FPG $(P=0.052)$ or PSI $(P=0.364)$ was found between groups. The change in ALT from baseline for the miglitol group was $8.40 \pm 7.20 \mathrm{U} / \mathrm{L}$ compared to $2.29 \pm 6.66 \mathrm{U} / \mathrm{L}$ for the placebo group $(P=0.009)$. For both groups, findings for ALT were similar between baseline and week 24 (visit 8) in terms of median, SD, and range; however, the mean value was significantly increased in the miglitol group, owing to a single patient with underlying chronic hepatitis and fatty liver.

Glycemic control in the ITT population, as measured by HbA1c level, showed significant improvement in both the miglitol and placebo groups compared to baseline (week 0 ) after 12 weeks $(P<0.01), 16$ weeks $(P<0.01), 20$ weeks $(P<0.001)$, and 24 weeks $(P<0.001)$ (Fig. 2$)$. In addition, the decrease from baseline to 24 weeks in $\mathrm{HbA} 1 \mathrm{c}$ was significantly higher in the miglitol group than in the placebo group $(P<0.001)$.

Among the 105 patients, $49(94.2 \%)$ in the miglitol group and $42(79.3 \%)$ in the placebo group experienced at least 1 adverse event during the study period. A total of 59 and 39 adverse events occurred in the miglitol and placebo groups, respectively. Table 3 shows the most frequent adverse events, which included abdominal discomfort, diarrhea, hypoglycemia, and other. Patients in the miglitol group reported other adverse events significantly more often than did those in the placebo group $(P=0.036)$. No major episodes requiring external assistance were reported. No clinically significant changes in any of the hematologic or clinical biochemistry variables were identified, and all changes were within normal range, with the exception that
Fig. 1 Progress of patients throughout the 24-week trial period

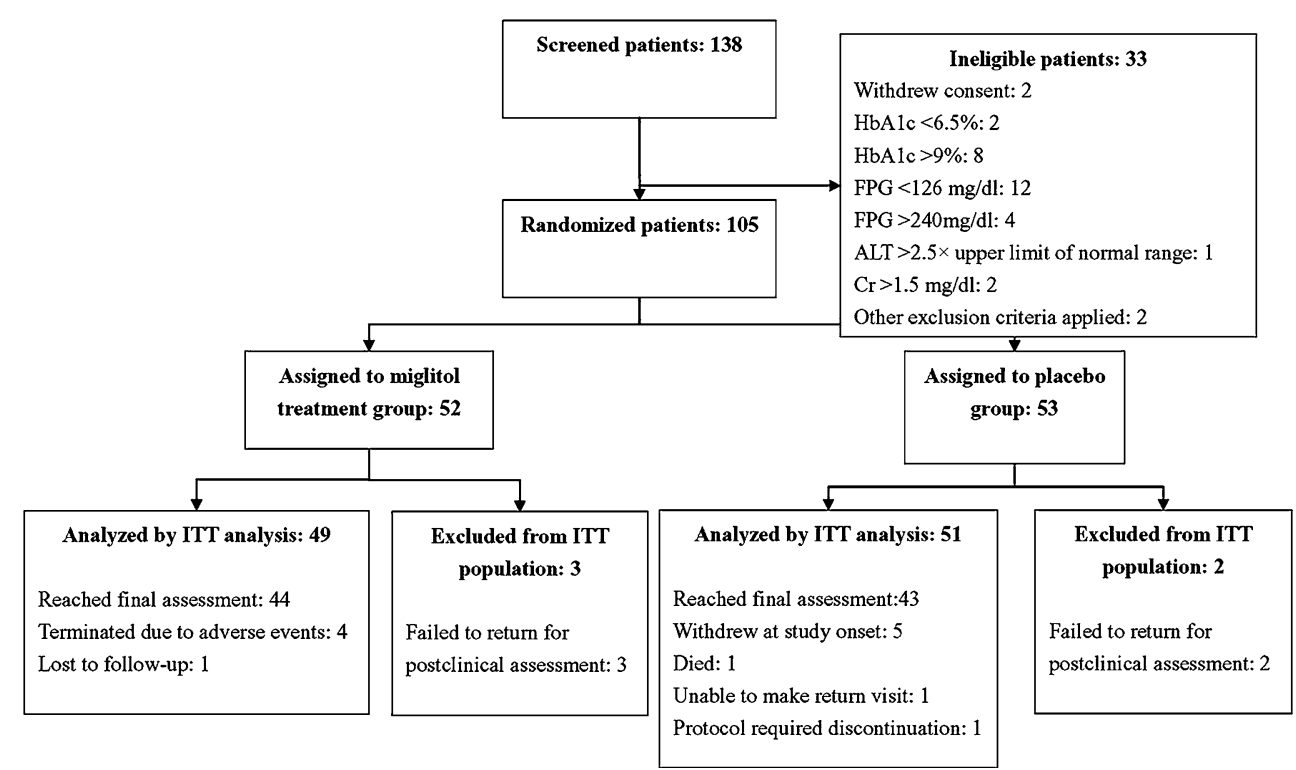


Table 1 Demographic and baseline clinical characteristics in 105 randomized patients with type 2 diabetes

\begin{tabular}{lllll}
\hline Characteristic & Total $(N=105)$ & Miglitol $(n=52)$ & Placebo $(n=53)$ & $P$ value \\
\hline Age $($ years $)$ & $58.7 \pm 10.5$ & $58.4 \pm 10.5$ & $59.0 \pm 10.7$ & $18 / 35$ \\
Gender $(\mathrm{M} / \mathrm{F})$ & $41 / 64$ & $23 / 29$ & $69.2 \pm 10.6$ & 0.759 \\
Body weight $(\mathrm{kg})$ & $68.2 \pm 10.5$ & $67.2 \pm 10.3$ & $162.8 \pm 8.4$ & 0.319 \\
Height $(\mathrm{cm})$ & $162.8 \pm 8.2$ & $162.8 \pm 8.1$ & $26.1 \pm 2.9$ & 0.978 \\
BMI $\left(\mathrm{kg} / \mathrm{m}^{2}\right)$ & $25.7 \pm 3.0$ & $25.3 \pm 3.1$ & $11 \pm 0.77$ \\
HbA1c $(\%)$ & $8.13 \pm 0.74$ & $8.14 \pm 0.72$ & $155.10 \pm 30.57$ \\
FPG $(\mathrm{mg} / \mathrm{dL})$ & $160.06 \pm 29.69$ & $165.13 \pm 28.17$ & $220.80 \pm 71.12$ \\
PPG $(\mathrm{mg} / \mathrm{dL})$ & $233.52 \pm 66.42$ & $246.76 \pm 58.98$ & $43.09 \pm 32.38$ \\
PSI $(\mu \mathrm{U} / \mathrm{mL})$ & $41.85 \pm 32.28$ & $40.59 \pm 32.44$ & 0.893 \\
\hline
\end{tabular}

Data are presented as mean \pm standard deviation (SD) for all variables, with the exception of gender $(n)$

$P$ values were determined by 2-sample $t$ test, with the exception of PSI, which was assessed by nonparametric Wilcoxon rank-sum test $B M I$ Body mass index, $H b A l c$ glycated hemoglobin, $F P G$ fasting plasma glucose, $P P G$ postprandial plasma glucose, $P S I$ postprandial serum insulin

Table 2 Changes in efficacy endpoints from baseline by study group in the intention-to-treat population $(n=100)$

\begin{tabular}{lccc}
\hline Characteristics $^{\mathrm{a}, \mathrm{b}}$ & Miglitol $(n=49)$ & Placebo $(n=51) P$ value $^{\mathrm{c}}$ \\
\hline HbA1c $(\%)$ & $-0.85 \pm 0.12$ & $-0.19 \pm 0.11$ & $<0.001^{\mathrm{d}}$ \\
FPG $(\mathrm{mg} / \mathrm{dL})$ & $-13.44 \pm 5.48$ & $-0.20 \pm 3.91$ & 0.052 \\
PPG $(\mathrm{mg} / \mathrm{dL})$ & $-44.8 \pm 10.43$ & $14.07 \pm 9.47$ & $<0.001^{\mathrm{d}}$ \\
PSI $(\mu \mathrm{U} / \mathrm{mL})$ & $-4.53 \pm 3.91$ & $-3.78 \pm 5.34$ & 0.364 \\
AST $(\mathrm{SGOT}, \mathrm{U} / \mathrm{L})$ & $1.96 \pm 3.66$ & $-1.5 \pm 1.23$ & 0.888 \\
ALT $(\mathrm{SGPT}, \mathrm{U} / \mathrm{L})$ & $8.40 \pm 7.20$ & $2.29 \pm 6.66$ & $0.009^{\mathrm{d}}$ \\
HDL-C & $-0.50 \pm 1.67$ & $-2.91 \pm 1.39$ & 0.861 \\
LDL-C & $0.81 \pm 3.84$ & $-9.36 \pm 2.90$ & $0.037^{\mathrm{d}}$ \\
TG & $-31.15 \pm 11.16$ & $-2.96 \pm 12.97$ & 0.156 \\
Total cholesterol & $0.042 \pm 4.11$ & $-7.0 \pm 3.31$ & 0.185 \\
\hline
\end{tabular}

a Data were presented as mean \pm standard error (SE)

b HbA1c Glycated hemoglobin, FPG fasting plasma glucose, $P P G$ postprandial plasma glucose, $P S I$ postprandial serum insulin, AST aspartate aminotransferase, $A L T$ alanine aminotransferase, $H D L$ highdensity lipoprotein cholesterol, $L D L$ low-density lipoprotein cholesterol, $T G$ triglyceride

c $P$ values for estimation of differences between change in miglitol and placebo groups from baseline were assessed by 2-sample $t$ test for $\mathrm{HbA1c}, \mathrm{FPG}$, and PPG and by nonparametric Wilcoxon rank-sum test for PSI, AST, ALT, HDL, LDL, and TG

${ }^{d} P<0.05$ indicated that the change from baseline of efficacy endpoint was significantly different between the miglitol and placebo treatment groups

4 patients ( 2 in the miglitol group, and 2 in the placebo group) had elevated liver enzymes at the end of the study. However, these same patients already had abnormal liver enzyme levels at baseline. There were no significant differences between treatment groups with respect to vital signs or results of physical examination, urinalysis, or electrocardiography.

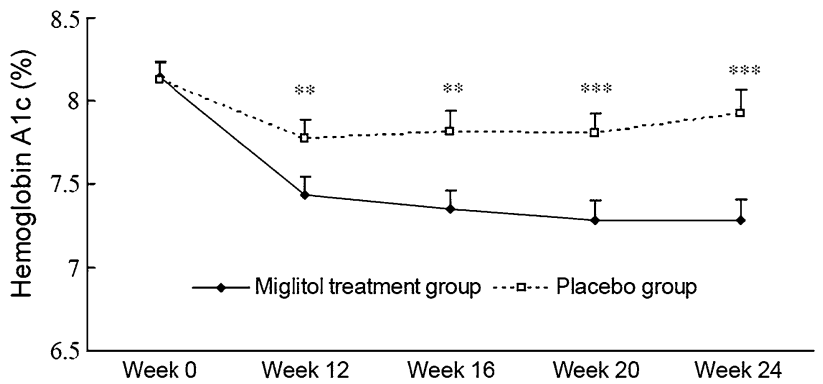

Fig. 2 Glycated hemoglobin levels for each study group in the intentto-treat population $(N=100)$ during the 24-week trial period. $* * P<0.01, * * * P<0.001$

Table 3 Most frequent adverse events by study group in the safety population $(n=105)$

\begin{tabular}{lccc}
\hline Adverse event* & $\begin{array}{l}\text { Miglitol } \\
(n=59)\end{array}$ & $\begin{array}{l}\text { Placebo } \\
(n=39)\end{array}$ & $P$ value \\
\hline Abdominal discomfort $^{\dagger}$ & $10(16.95)$ & $2(5.13)$ & 0.084 \\
Diarrhea $^{\ddagger}$ & $20(33.90)$ & $7(17.95)$ & 0.117 \\
Hypoglycemia $^{*}$ & $20(33.90)$ & $17(43.59)$ & 0.333 \\
Other $^{*}$ & $9(15.25)$ & $13(33.33)$ & $0.036^{\S}$
\end{tabular}

* Data are presented as $n(\%)$ of all adverse events compared with

${ }^{*}$ Chi-square or ${ }^{\dagger}$ Fisher exact test

$\S P$ values $<0.05$ indicate that the incidence of a specific adverse event was significantly different between the 2 groups

\section{Discussion}

The objective of the present study was to investigate the efficacy and tolerability of miglitol in combination with sulfonylureas, compared to sulfonylurea monotherapy, for the improvement of glycemic control in Chinese outpatients with type 2 diabetes mellitus inadequately controlled 
by diet and sulfonylurea treatment. The primary endpoint was change from baseline in HbA1c concentration at week 24. Results showed a greater than fourfold difference between the change in HbA1c from baseline in the miglitol group compared with the placebo group, with the difference between the 2 groups reaching statistical significance at week $12(P<0.01)$. Clinically significant effects of miglitol treatment were found from weeks 12 to 24 . This result corresponded well with decreases in $\mathrm{HbA} 1 \mathrm{c}$ reported in another study of miglitol adjuvant therapy [18].

The HbAlc concentration reflects long-term glycemic control [19], and a decrease in HbAlc results in a reduced risk of microvascular adverse events [5]. A major problem in the use of oral antidiabetic drugs, such as sulfonylureas, is the gradual decrease in the ability of these drugs to satisfactorily control blood glucose level [20]. Pharmacologic agents are available that modify primarily the PPG level to reduce serum HbA1c [21, 22]. $\alpha$-Glucosidase inhibitors produce an antihyperglycemic effect and do not induce weight gain, a common problem encountered with sulfonylureas and insulin [23]. A recent meta-analysis of 41 randomized trials examined the efficacy of $\alpha$-glucosidase inhibitors in patients with type 2 diabetes and showed no evidence of a beneficial effect on morbidity or mortality [24]. However, statistically significant effects on HbAlc (by acarbose), FPG (by miglitol), postload glucose, insulin level, and BMI (by acarbose) were found. The study found no effects on PSI or lipids and only minor effects on body weight.

The clinical significance of the regulation of postprandial hyperglycemia in reducing the risk of microvascular and macrovascular complications has been established in several epidemiologic studies [25-27]. Results of the present study are of interest because the PPG level was significantly decreased in the miglitol group compared with the placebo group $(P<0.001)$. With respect to lipid variables, we found no significant differences between groups in the present study, similar to previous results [24].

The major adverse effects of $\alpha$-glucosidase inhibitors, such as acarbose, include gastrointestinal symptoms; these arise mainly from the fermentation of undigested carbohydrates by colonic bacteria [28]. In contrast, miglitol is absorbed systemically, but is not metabolized, and is excreted into the urine within a relatively short period of time. Consequently, systemic adverse effects are not anticipated. Indeed, no systemic adverse effects occurred in the present study. There was also no incidence of severe hypoglycemia. The most common complaints in this study were mild hypoglycemia, diarrhea, and abdominal discomfort, resulting in premature termination of miglitol treatment by 4 patients. Approximately half of the patients in the miglitol group and $15.7 \%$ of the patients in the placebo group experienced these symptoms.
Several case reports from Europe and Japan have indicated that acarbose, a commonly prescribed $\alpha$-glucosidase inhibitor, can result in severe, but reversible, hepatotoxicity, as indicated by markedly increased levels of AST and ALT [29-31]. In contrast, treatment with miglitol at the dosages used in the present study increased AST and ALT to a much lesser extent $(\leq 1.8$ times to upper limit of normal) [17]. Because of the relatively high prevalence of hepatitis B and hepatitis $\mathrm{C}$ in Asian countries, the use of miglitol may prove to be a better choice compared to acarbose with respect to minimizing hepatic effects.

The small number of cases and the relatively short, 24-week study period are potential limitations of the present study. Future large-scale studies are needed to assess the long-term cardiovascular and glucose-control effects of miglitol.

\section{Conclusions}

Results of the present study indicate that miglitol improved PPG level and glycemic control, as reflected by decreased HbA1c concentration in Chinese patients with type 2 diabetes mellitus. Miglitol was well tolerated, with no unusual changes in safety profiles, with the exception of abdominal discomfort during the 24 -week treatment period. Therefore, miglitol may be a useful adjuvant therapy for Chinese patients with type 2 diabetes inadequately controlled by diet and sulfonylurea treatment.

Acknowledgments This study was supported in part by Tri-Service General Hospital, Taipei, Taiwan (TSGH-C96-5-S03) and by Standard Chem. \& Pharm. Co., Ltd. The authors would like to acknowledge the contributions of the following co-principle investigators: Dr. Feng-Hsuan Liu and Dr. Hung-Yu Chang, Division of Endocrinology and Metabolism, Chang Gung Memorial Hospital, Chang Gung University College of Medicine, Taipei, Taiwan; Dr. Jenn-Kuen Lee, Dr. Chih-Chen Lu, and Dr. Chun-Chin Sun, Division of Endocrinology and Metabolism, Kaohsiung Veterans General Hospital, Kaohsiung, Taiwan; and Dr. Chieh-Hua Lu and Dr. Chih-Tsueng He, Division of Endocrinology and Metabolism, Tri-Service General Hospital, National Defense Medical Center, Taipei, Taiwan.

Conflict of interest The authors declare no financial interests.

Open Access This article is distributed under the terms of the Creative Commons Attribution Noncommercial License which permits any noncommercial use, distribution, and reproduction in any medium, provided the original author(s) and source are credited.

\section{References}

1. US Department of Health and Human Services, Centers for Disease Control and Prevention (2005) National Diabetes Fact Sheet: General Information and National Estimates on Diabetes 
in the United States. Atlanta, GA, Centers for Disease Control and Prevention

2. Resnick HE, Foster GL, Bardsley J et al (2006) Achievement of American Diabetes Association clinical practice recommendations among US adults with diabetes, 1999-2002: the National Health and Nutrition Examination Survey. Diabetes Care 29:531537

3. Lin RS, Lee WC (1992) Trends in mortality from diabetes mellitus in Taiwan, 1960-1988. Diabetologia 35:973-979

4. Tseng CH (2004) Mortality and causes of death in a national sample of diabetic patients in Taiwan. Diabetes Care 27:16051609

5. UK Prospective Diabetes Study (UKPDS) Group (1998) Intensive blood-glucose control with sulphonylureas or insulin compared with conventional treatment and risk of complications in patients with type 2 diabetes (UKPDS 33). Lancet 352:837-853

6. Praet SF, van Loon LJ (2009) Exercise therapy in type 2 diabetes. Acta Diabetol 46:263-278

7. Zanuso S, Jimenez A, Pugliese G et al (2010) Exercise for the management of type 2 diabetes: a review of the evidence. Acta Diabetol 47:15-22

8. Vijay SK, Mishra M, Kumar H et al (2009) Effect of pioglitazone and rosiglitazone on mediators of endothelial dysfunction, markers of angiogenesis and inflammatory cytokines in type-2 diabetes. Acta Diabetol 46:27-33

9. Doggrell SA (2008) Clinical trials with thiazolidinediones in subjects with Type 2 diabetes-is pioglitazone any different from rosiglitazone? Expert Opin Pharmacother 9:405-420

10. Lund SS, Tarnow L, Frandsen $M$ et al (2009) Combining insulin with metformin or an insulin secretagogue in non-obese patients with type 2 diabetes: 12 month, randomised, double blind trial. BMJ 339:b4324

11. Pala L, Monami M, Lamanna C et al (2009) Failure to metformin and insulin secretagogue monotherapy: an observational cohort study. Acta Diabetol. Mar 17. [Epub ahead of print]

12. Campbell LK, White JR, Campbell RK (1996) Acarbose: its role in the treatment of diabetes mellitus. Ann Pharmacother 30:12551262

13. Bischoff H (1994) Pharmacology of alpha-glucosidase inhibition. Eur J Clin Invest 24(Suppl 3):3-10

14. Holman RR, Steemson J, Turner RC (1991) Post-prandial glycaemic reduction by an alpha-glucosidase inhibitor in type 2 diabetic patients with therapeutically attained basal normoglycaemia. Diabetes Res 18:149-153

15. Saunier B, Kilker RD Jr, Tkacz JS et al (1982) Inhibition of $\mathrm{N}$-linked complex oligosaccharide formation by 1-deoxynojirimycin, an inhibitor of processing glucosidases. J Biol Chem 257:14155-14161

16. Segal P, Feig PU, Schernthaner G et al (1997) The efficacy and safety of miglitol therapy compared with glibenclamide in patients with NIDDM inadequately controlled by diet alone. Diabetes Care 20:687-691

17. Johnston PS, Coniff RF, Hoogwerf BJ et al (1994) Effects of the carbohydrase inhibitor miglitol in sulfonylurea-treated NIDDM patients. Diabetes Care 17:20-29

18. Chiasson JL, Naditch L (2001) Miglitol Canadian University Investigator Group. The synergistic effect of miglitol plus metformin combination therapy in the treatment of type 2 diabetes. Diabetes Care 24:989-994

19. Coniff RF, Shapiro JA, Robbins D et al (1995) Reduction of glycosylated hemoglobin and postprandial hyperglycemia by acarbose in patients with NIDDM. A placebo-controlled dosecomparison study. Diabetes Care 18:817-824

20. Holman RR, Cull CA, Turner RC (1999) A randomized doubleblind trial of acarbose in type 2 diabetes shows improved glycemic control over 3 years (UK Prospective Diabetes Study 44). Diabetes Care 22:960-964

21. American Diabetes Association (2006) Standards of medical care in diabetes-2006. Diabetes Care 29(1):S4-S42

22. Yamasaki Y, Katakami N, Hayaishi-Okano R et al (2005) alphaGlucosidase inhibitor reduces the progression of carotid intimamedia thickness. Diabetes Res Clin Pract 67:204-210

23. Turner RC (1998) The UK prospective diabetes study. A review. Diabetes Care 21(Suppl 3):C35-C38

24. van de Laar FA, Lucassen PL, Akkermans RP et al (2005) Alphaglucosidase inhibitors for patients with type 2 diabetes: results from a Cochrane systematic review and meta-analysis. Diabetes Care 28:154-163

25. Tominaga M, Eguchi H, Manaka $H$ et al (1999) Impaired glucose tolerance is a risk factor for cardiovascular disease, but not impaired fasting glucose. The Funagata Diabetes Study. Diabetes Care 22:920-924

26. Chiasson JL, Josse RG, Gomis R et al (2002) Acarbose for prevention of type 2 diabetes mellitus: the STOP-NIDDM randomised trial. Lancet 59:2072-2077

27. DECODA Study Group; International Diabetes Epidemiology Group (2002) Cardiovascular risk profile assessment in glucoseintolerant Asian individuals - an evaluation of the World Health Organization two-step strategy: the DECODA Study (Diabetes Epidemiology: Collaborative Analysis of Diagnostic Criteria in Asia). Diabetes Med 19:549-557

28. Balfour JA, McTavish D (1993) Acarbose. An update of its pharmacology and therapeutic use in diabetes mellitus. Drugs 46:1025-1054

29. Diaz-Gutierrez FL, Ladero JM, Diaz-Rubio M (1998) Acarboseinduced acute hepatitis. Am J Gastroenterol 93:481

30. Andrade RJ, Lucena M, Vega JL et al (1998) Acarbose-associated hepatotoxicity. Diabetes Care 21:2029-2030

31. Fujimoto Y, Ohhira M, Miyokawa N et al (1998) Acarboseinduced hepatic injury. Lancet 351:340 